\title{
Opportunities of in situ TEM for measuring voltage-driven microstructural changes in memristive devices
}

Frances Ross ${ }^{1}$, Baoming Wang ${ }^{2}$, Teodor Todorov ${ }^{3}$ and John Rozen ${ }^{3}$

${ }^{1}$ MIT, United States, ${ }^{2}$ Massachusetts Institute of Technology, Cambridge, Massachusetts, United States, ${ }^{3}$ IBM T. J. Watson Research Center, United States

Synaptic elements that are capable of reversible, multilevel analog switching may form the basis of future neuromorphic computing devices that have the potential to exceed the practical limits of current digital technology. The field of neuromorphic computing can benefit greatly from the ability to see the individual synaptic elements as they switch between states while simultaneously measuring their electrical parameters. In situ TEM biasing has therefore become one of several key experimental tools used to image switching phenomena in individual devices [1]. Here we will discuss how sample design can be optimized for identifying mechanisms and probing reliability and aging phenomena, illustrating with measurements made on devices based on a new type of copper ion conductor.

Many types of synaptic elements display stochastic switching processes, making it beneficial if the entire device is accessible for imaging during the in situ biasing experiment. Figure 1 shows a three-terminal synaptic element that has been fabricated in a plan view geometry such that the entire device area is electron transparent. The device operates via the motion of copper ions through polycrystalline $\mathrm{Cu}_{16} \mathrm{Rb}_{4} \mathrm{Cl}_{13} \mathrm{I}_{7}$. This unlikely sounding material has the highest room temperature conductivity of all reported solid electrolytes, $0.34 \mathrm{~S} / \mathrm{cm}$ [2]. Writing consists of applying a voltage between gate and source/channel/drain to force copper to migrate through the electrolyte that connects the gate and the channel. After writing, reading consists of applying a small voltage between source and drain to measure the channel resistance. In principle, as a layer of copper is added or subtracted from the channel by positive or negative write pulses, the channel resistance should change. The resistivity is therefore sensitive to the history of write pulses applied to the device, as required for a memristive synaptic element [3].

In situ observations using this sample geometry display a range of physical phenomena taking place within the electrolyte and at its interfaces with the electrodes. Application of voltage pulses forms dramatic bright bands that migrate across the electrolyte during biasing, while the current and voltage show memristive behavior. EDX mapping shows that these bands are copper deficient and that their formation is associated with a grain coarsening process. The bands can be healed by reverse biasing, but when driven at too high a voltage copper does not "refill" the structure and instead iodine evaporates to form voids, an irreversible degradation mechanism. We model these structural changes in terms of local regions of higher resistivity created by motion of copper ions, aiming to define critical limits for reversible operation. Device operation also involves structural changes at the electrolyte/channel interface. For channels made of Pt, copper deposits to form nanoparticles and dendrites, whereas carbon channels enable intercalation without solid copper formation. The difference, based on copper solubility and diffusion within the electrode, provides guidance for device design. 
The lateral geometry in Figure 1 is less well suited for devices in which the electrolyte is less conductive and is present in the device as a thinner film. These include materials such as $\mathrm{HfO}_{2}, \mathrm{WO}_{3}$ and $\mathrm{CeO}_{2}$ in which ionic transport phenomena can be used to create neuromorphic-like device configurations $[4,5]$. We discuss strategies for cross-sectional device geometry, particularly measuring and accounting for the parallel conduction pathways through surface layers. Based on these measurements, quantitative information on the spatial distribution, reproducibility and aging phenomena associated with ion motion can be achieved in devices that are electron transparent yet show similar electrical characteristics to bulk devices. We finally discuss the inclusion of additional parameters such as heating and mechanical stimuli, suggesting exciting future possibilities for in situ TEM experiments to help engineer improved elements for neuromorphic computing [6].
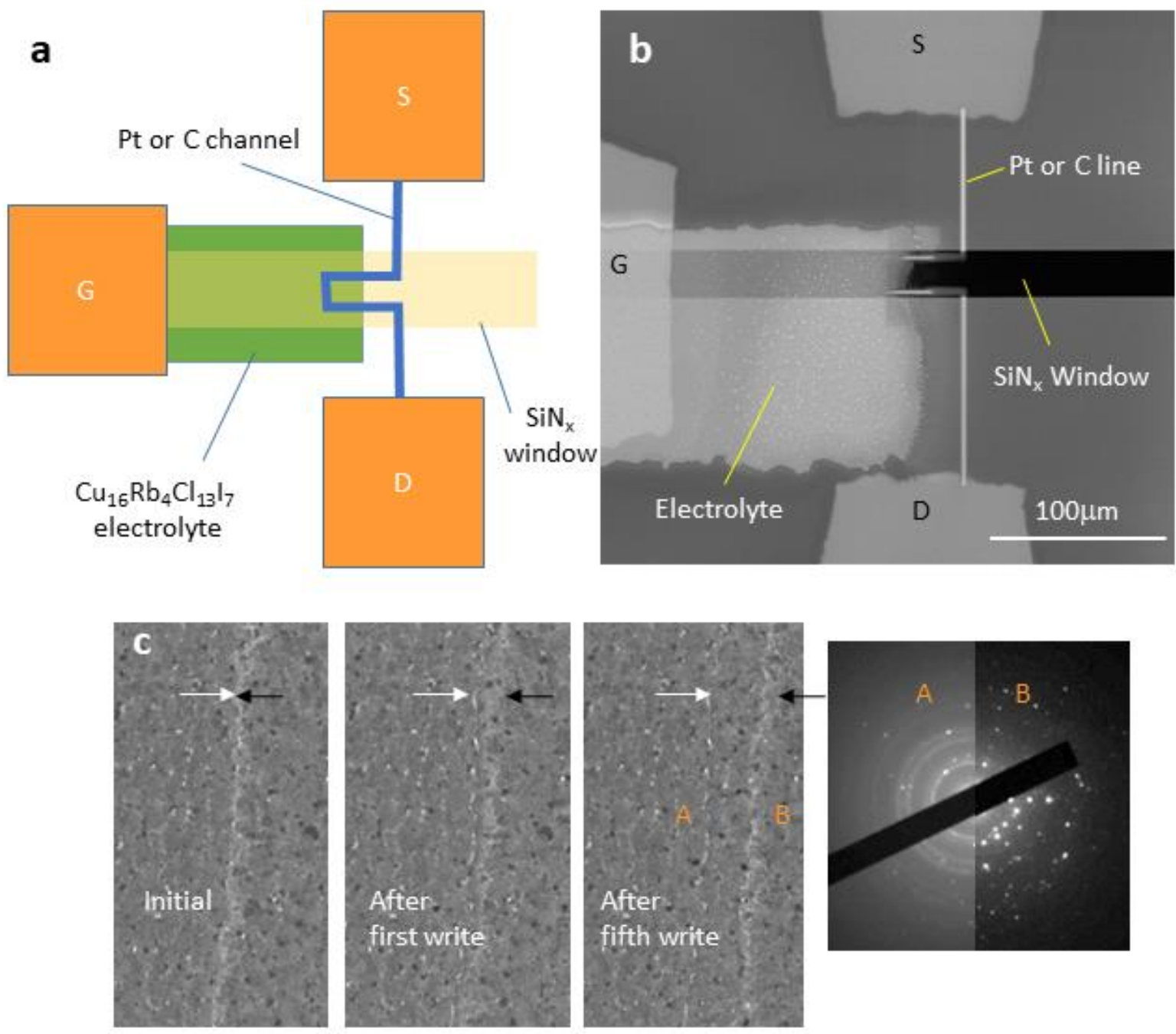

Figure 1. Figure 1. (a) Schematic and (b) SEM image of a synaptic element made of a copper ionic conductor designed to enable in situ observation. The device is fabricated on a commercial silicon nitride window chip and wire bonded to a sample holder with electrical feedthroughs. Images are recorded at up 
to 100 frames per second while controlling current and voltage. Note that the device can be designed such that the entire channel region is visible. (c) Image series extracted from a video showing morphological changes within the electrolyte near the channel during writing with pulses of $1 \mathrm{uA}, 1 \mathrm{~s}$ on $/ 4 \mathrm{~s}$ off. A bright band (black arrow) is visible that moves across the electrolyte as the structure converts from larger to finer grained. This brighter region represents lower material density and can therefore be related to the local depletion of copper ions from the structure.

\section{References}

[1] W. Sun, B. Gao, M. Chi., Q. Xia, J. J. Yang, H. Qian and H. Wu, Understanding memristive switching via in situ characterization and device modeling. Nat Commun 10, 3453 (2019).

[2] T. Takahashi, O. Yamamoto, S. Yamada and S. Hayashi, High Copper Ion Conductivity of the System $\mathrm{CuCl}$ - CuI - RbCl, J. EIectrochem. Soc. 126, 1654 (1979).

[3] T. Todorov, T. Ando, F. M. Ross, J. A. Ott, J. Tang, D. Bishop, J. Collins, V. Narayanan and J. Rozen, Copper-Based 3-Terminal Synaptic Cell with Multiple Resistance Levels, Electrochem. Soc. Meeting Abstracts 23, 1165 (2019).

[4] S. Kim et al., "Metal-oxide based, CMOS-compatible ECRAM for Deep Learning Accelerator," in 2019 IEEE International Electron Devices Meeting (IEDM), 35.7 .1 (2019).

[5] R. Waser, R. Dittmann, G. Staikov, and K. Szot, "Redox-Based Resistive Switching Memories Nanoionic Mechanisms, Prospects, and Challenges," Adv. Mater., 21, 2632 (2009).

[6] This work was partially supported by the MIT-IBM Watson AI Lab and made use of facilities and instrumentation supported by NSF through the Massachusetts Institute of Technology Materials Research Science and Engineering Center DMR-1419807, as well as facilities at MIT.nano. 